\title{
Aggregation of Fuzzy Classifiers Using Coupled Map Lattices
}

\author{
Jonatan Gómez and Elizabeth León
}

\begin{abstract}
This paper proposes a technique for aggregating a group of fuzzy classifiers using a coupled map lattice. First a the training data set is divided into several disjoint groups. Each group is used for training a classifier using a fuzzy classification technique. Then, each fuzzy classifier is associated to one site in a coupled map lattice. In order to predict the class of a given data sample, the sample is presented to each fuzzy classifier and the prediction is evolved using the dynamics properties of the coupled map lattice. The final prediction is the fuzzy voting of the classifiers after evolving them. The proposed approach is tested with several toy and real data sets in order to determine its performance.
\end{abstract}

\section{INTRODUCTION}

Classification is a supervised learning technique that takes labeled data samples and generates a model (classifier) that classifies new data samples in different predefined groups or classes [1]. Classification has been extensively studied in machine learning and data mining [1], [2], [3].

However, several empirical and theoretical analysis have shown that some classification techniques perform better than other techniques in different classification problems. Some research works have tried to develop different mechanisms by combining or aggregating different classification techniques [4]. The idea behind these mechanisms is to enhance the accuracy of the aggregated classifier since it is taking advantage of the local efficiency of each classifier being aggregated.

This paper proposes a technique for aggregating a group of fuzzy classifiers using a coupled map lattice. First a the training data set is divided into several disjoint groups. Each group is used for training a classifier using a fuzzy classification technique. Then, each fuzzy classifier is associated to one site in a coupled map lattice. In order to predict the class of a given data sample, the sample is presented to each fuzzy classifier and the prediction is evolved using the dynamics properties of the coupled map lattice. The final prediction is the fuzzy voting of the classifiers after evolving them. Section 2 gives an overview to the Coupled Map Lattices theory. Section 3 presents the proposed approach. Section 4 describes the set of fuzzy classification techniques aggregated with the proposed scheme. Section 5 presents the experiments performed and analyzes the results obtained. Section 6 draws some conclusions and future work.

\section{Coupled Map Lattices (CML)}

The Coupled Map Lattice (CML) theory grew out of studies on collective movements of coupled oscillators [5], [6] and can

Gómez and León are with the Department of Computer and Systems Engineering at Universidad Nacional de Colombia. e-mails: \{jgomezpe,eleonguz\}@ unal.edu.co

0-7803-9489-5/06/\$20.00/@2006 IEEE be seen as extensions of the concept of cellular automaton [7]. A CML can be defined in any $d$-dimensional space $(d=$ $1,2, .$.$) but in this paper, we consider only 2-dimensional CML$ of size $N \times M$.

A 2-D Coupled Map Lattice is a 2-dimensional lattice of size $N \times M$ such that each site $x(i, j)$ evolves according to equation 1 .

$x(i, j)_{t+1}=(1-\varepsilon) f\left(x_{t}(i, j)\right)+\frac{\varepsilon}{s\left(N_{x(i, j)}\right)} \sum_{y \in N_{x(i, j)}} f\left(y_{t}\right)$

where, $N_{x(i, j)}$ is a collection of sites which defines the neighborhood of site $x(i, j), s\left(N_{x(i, j)}\right)$ indicates the number of neighbors, $f$ is a non-linear function that determines the coupling value of a site, and $\varepsilon$ is a parameter that determines the coupling level or spatial correlation between sites. By varying the coupling parameter $\varepsilon$ and the structure of the neighborhood $N_{x(i, j)}$, it is possible to simulate systems that interact locally up to systems that interact globally [6].

Several non-linear functions have been used into a CML. In the following discussion we use the well-known logistic function:

$$
f(x)=1-\alpha x^{2}
$$

where, $0 \leq \alpha \leq 2$ is a suitable parameter that allows one to modify the dynamics of the system and introduce chaotic behavior into the CML. In order to introduce chaos to the CML, $\alpha$ should be set to a value higher than 1.82 .

Coupled map lattices have been used for simulating natural dynamic processes such as fluid dynamics. Moreover, Coupled Map Lattices can be seen as a computational model.

\section{PROPOSED APPROACH}

We organize the fuzzy classifiers in a two dimensional Coupled Map Lattice (CML) of size $N \times M$ where the neighborhood of site $x(i, j)$ is defined according to equation $3^{1}$.

$$
N_{x(i, j)}=\{x(i, j+1), x(i, j-1), x(i-1, j), x(i-1, j)\}
$$

Figure 1 shows the proposed Cell Organized Recognition Algorithms (CORAL) neighborhood definition for a CML of size $3 \times 3$.

The state of a site $x(i, j)$ on any time $t \geq 0$ indicates the confidence degree that such site has on classifying a data

${ }^{1}$ Sites that are on the border are connected with sites that are in the opposite border in the same row (column). 


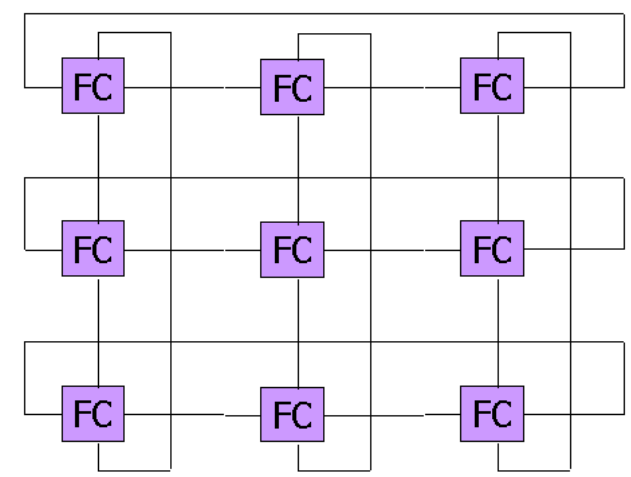

Fig. 1. CORAL neighborhood definition for a CML of size $3 \times 3$.

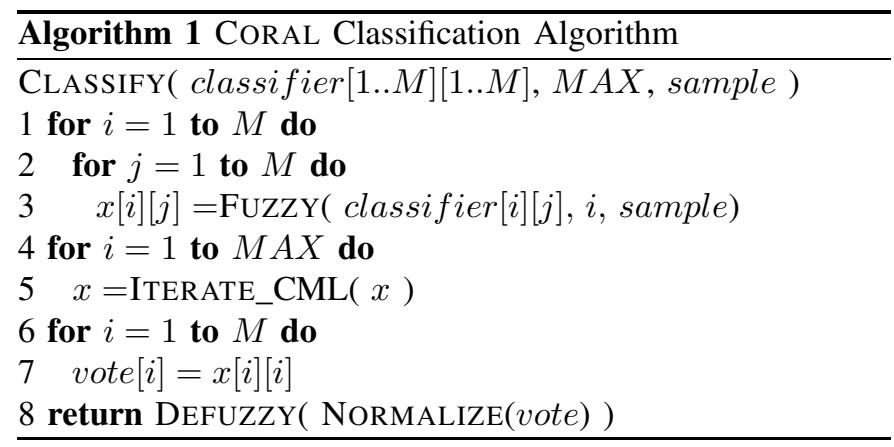

sample sample into the negative class. The initial state of site $x(i, j)$ is defined as the membership degree of the sample to the negative class generated by the classifier associated to such site, see Equation 4.

$$
\left.x_{0}(i, j)=\mu_{-, \text {classifier }}, \text { (sample }\right)
$$

Now, the state of a site $x(i, j)$ evolves on time according to equation 5 :

$$
\begin{aligned}
x_{t+1}(i, j)= & (1-\varepsilon) x_{t}(i, j)+ \\
& \frac{\varepsilon}{4}\left[f\left(x_{t}(i, j-1)\right)+f\left(x_{t}(i, j+1)\right)+\right. \\
& \left.\left.f\left(x_{t}(i-1, j)\right)+f\left(x_{t}(i+1, j)\right)\right]\right)
\end{aligned}
$$

where $f(x)$ is the logistic function, see equation 2. Finally, the classification of a data sample is determined by a de-fuzzy operator that is applied to the state of the sites in the diagonal after some number of iterations $M A X$ of the CML. Algorithm 1 shows the CORAL classification.

\section{Classification Techniques Aggregated with CORAL}

\section{A. Evolution of Fuzzy Rules}

Due to high interpretability of fuzzy rule based classifiers (FRBC) and the ability of evolutionary algorithms (EA) to find good solutions, some research work has focused on developing evolutionary techniques for generating FRBC [8], [9], [10], [11], [12], [13]. These techniques receive the name of Genetic Fuzzy Rule Based Systems (GFRBS) [14], [15]. Of several GFRBS approaches proposed, all of them differ from each other in at least one of the following aspects: number of fuzz rules that each individual encodes, type of rule expression encoded by an individual, and scope of the evolutionary process [8], [16], [14].

In order to evolve a FRBC, the approach proposed by Gomez in [17], [18] was applied. In this way, an evolutionary algorithm (EA) is executed for evolving a fuzzy rule for a twoclass classification problem. The EA takes as input the training data set, applies the evolutionary strategies, and returns one fuzzy rule. Such a rule has the form : $R$ : IF condition THEN data is positive. A data sample is classified as positive with the truth-value (TV) of the fuzzy rule $R$ and classified as negative with TV equal to the fuzzy negation of the TV of $R$. The best individual of the population, according to the fitness value, will determine the fuzzy rule that will be used for discriminating between the two classes under consideration.

1) Fuzzy Rule Encoding: Since we are dealing with a two class problem, it is not necessary to encode the class that the fuzzy rule is discriminating (it is always the positive class). Only the fuzzy expression that corresponds with the condition part of the fuzzy rule is encoded. Gomez used a heap encoding scheme by allowing the EA to evolve the fuzzy sets associated with the atomic conditions [18]. The linear representation of such heap trees is defined as a list of genes, each gene encoding an atomic expression (defined as fuzzy_variable [is/not] fuzzy_set) and a logic operator (and or $o r)$. The logic operator encoded in the last gene is not taken into account because the number of logic operators is one less than the number of atomic expressions, see Figure 2.

We use $\left\lceil\log _{2}(m)\right\rceil$ bits for encoding $m$ possible attributes, one bit for the membership relation $(\in / \notin)$ and one bit for the logic operator $(\wedge / \vee)$. The number of bits used for representing the fuzzy set depends on the scope of the evolutionary process; in our case with fuzzy set tuning.

Instead of encoding the index of a predefined fuzzy set into each gene as proposed by Gomez et al. in [8] where $\left\lceil\log _{2}(m)\right\rceil$ bits are used for representing $m$ predefined fuzzy sets, a set of parameters defining a fuzzy set can be encoded into each gene and allow the EA to tune it. Isosceles triangular fuzzy sets can be tuned by encoding two values, the points defining the base of the triangle [19]. Gaussian shaped fuzzy sets can be defined by encoding two values, the median and the standard deviation. In this paper, the fuzzy set tuning is restricted to trapezoidal fuzzy sets defined by two parameters. The attribute space is divided into $m$ regions of the same length ( $m$ is a parameter given by the user). This division generates $m+1$ control points, see Figure 3.a. Given two control points, $x$ and $y(x \leq y)$, it is possible to define the trapezoidal fuzzy set: $\left(\max \left\{0, \frac{x-1}{m}\right\}, \frac{x}{m}, \frac{y}{m}, \min \left\{1, \frac{y+1}{m}\right\}\right)$, see Figure $3 . b^{2}$.

Therefore, $2\left\lceil\log _{2}(m+1)\right\rceil$ bits are used for representing the two control points of the trapezoidal fuzzy set.

2) Genetic Operators: The following are the genetic operators that we will used with the proposed encoding:

- Variable Length Simple Point Crossover (VLSPX). Given two chromosomes $A=a_{1} a_{2} . . a_{n}$ and $B=$ $b_{1} b_{2} . . b_{m}, n$ and $m$ are the size in bits of $A$ and $B$ respectively, the VLSPX selects a random point $k$ in the interval

${ }^{2}$ It will define a triangular fuzzy set instead of a trapezoidal if $x=y$. 


\begin{tabular}{|c|c|c|c|c|c|c|c|c|}
\hline \multicolumn{4}{|c|}{ Gene $_{1}$} & $\ldots$ & \multicolumn{2}{|c|}{ Gene $_{n-1}$} & \multicolumn{2}{|c|}{ Gene $_{n}$} \\
\hline & Atom $_{1}$ & & $O p_{1}$ & $\ldots$ & Atom $_{n-1}$ & $O p_{n-1}$ & Atom $_{n}$ & $*$ \\
\hline var & $\in / \notin$ & set & $\wedge / \vee$ & $\ldots$ & & & & $*$ \\
\hline
\end{tabular}

Fig. 2. Linear Representation of heaps.

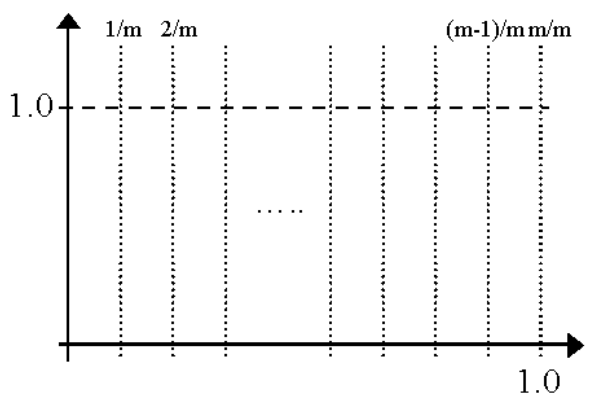

(a) Division of the space in $\mathrm{m}$ intervals

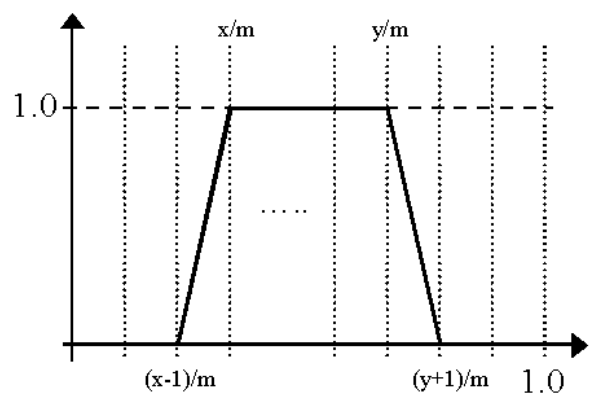

(b) Two control points trapezoid

Fig. 3. Tuning of Trapezoidal membership functions.

$[2, \min \{n, m\}-1]$, and generates two offspring $C=$ $a_{1} a_{2} . . a_{k} b_{k+1} . . b_{m}$ and $D=b_{1} b_{2} . . b_{k} a_{k+1} . . a_{n}$. When PFEs are encoded, it is possible that VLSPX does not only exchange genes but modifies one of them (if the crossover point is selected in the middle of such gene).

- Single Bit Mutation (SBM). Given a chromosome $A=$ $a_{1} a_{2} . . a_{k} . . a_{n}$, the SBM produces an offspring by flipping one random bit in it, $C=a_{1} a_{2} . . \overline{a_{k}} . . a_{n}$.

- Gene Addition (ADD). Given a chromosome $A=$ $a_{1} a_{2} . . a_{n}$ of $n$ genes, the ADD operator produces an offspring by generating a random gene $r$ and appending it to the end of the chromosome: $C=a_{1} a_{2} . . a_{n} r$.

- Gene Deletion (DEL). Given a chromosome $A=$ $a_{1} a_{2} . . a_{n}$ of $n \geq 2$ genes, the DEL operator produces an offspring by removing the last of the chromosome, $C=a_{1} a_{2} . . a_{n-1}$.

3) Fitness Function: The concept of fuzzy confusion matrix was introduced in [20] in order to determine the performance of an individual. Values in a confusion matrix (see table ??) correspond with the cardinality of intersection sets. For example, $P P$ is the number of positive samples that are classified (predicted) as positive, i.e., the cardinality of the set: Actual-Positive $\cap$ Predicted-Positive. These values can be calculated by using the membership function of the data samples to the actual and predicted data sets as:

$$
\begin{gathered}
P P=\sum_{i=1}^{n} \mu_{A}\left(d_{i}\right) \wedge \mu_{B}\left(d_{i}\right) \\
P N=\sum_{i=1}^{n} \mu_{C}\left(d_{i}\right) \wedge \mu_{B}\left(d_{i}\right)=\sum_{i=1}^{n} \overline{\mu_{A}\left(d_{i}\right)} \wedge \mu_{B}\left(d_{i}\right) \\
N P=\sum_{i=1}^{n} \mu_{A}\left(d_{i}\right) \wedge \mu_{D}\left(d_{i}\right)=\sum_{i=1}^{n} \mu_{A}\left(d_{i}\right) \wedge \overline{\mu_{B}\left(d_{i}\right)}
\end{gathered}
$$

$$
N N=\sum_{i=1}^{n} \mu_{C}\left(d_{i}\right) \wedge \mu_{D}\left(d_{i}\right)=\sum_{i=1}^{n} \overline{\mu_{A}\left(d_{i}\right)} \wedge \overline{\mu_{B}\left(d_{i}\right)}
$$

Where, $n$ is the number of samples used to test the classifier, $A$ is the actual (real) positive set, $B$ is the predicted positive set, $C$ is the actual negative set, $D$ is the predicted negative set, and $d_{i}$ is the $i$-th data record sample in the data set.

Notice that, for a two-class classification problem, one only needs to know the membership of a data sample to the actual data set and to the predicted membership value of the positive set.

Equations 6, 7, 8 and 9 can be extended to fuzzy sets and fuzzy rules. The degree of membership of the data sample to the actual positive set is given by the data sample label and the predicted membership to the positive set is calculated as the truth-value of the condition part of the fuzzy rule. The confusion matrix generated by using these extensions is called fuzzy confusion matrix. Performance metrics in table ?? can be generated from the fuzzy confusion matrix. Such new performance metrics will be called fuzzy performance metrics: (fuzzy accuracy, fuzzy true positives, etc ).

Since the goal of the evolutionary process is to generate a simple fuzzy rule that can discriminate the positive class from the negative, the fitness of an individual is defined by the fuzzy accuracy (FAC), and the fuzzy rule length (FRL), i.e. the number of atomic conditions defining the fuzzy rule. In this way, the optimization problem is a two-goal objective function: maximizing the FAC while minimizing the fuzzy rule length (FRL). Although there are several ways to deal with multi-goal objective functions, in this work, the weighted sum technique was used. Therefore, the fitness of an individual is calculated using equation 10 .

$$
\begin{array}{ll}
\operatorname{fitness}(R) & =w * F A C(R)+(1-w) *\left(1-\frac{F R L(R)}{M}\right) \\
F A C(R) & =\frac{P P(R)+N N(R)}{P P(R)+P N(R)+N P(R)+N N(R)}
\end{array}
$$


Here, $w$ is the weight associated with the fuzzy accuracy reached by the individual and $M$ is the maximum number of atomic expressions defining a fuzzy rule.

4) Rule Extraction: The best individual of the population, according to the fitness value, will determine the fuzzy rule that will be used for discriminating between the two classes under consideration.

\section{B. RAIN: Data Clustering using Randomized Interaction of Data Points}

In [21], Gomez et al developed a robust clustering technique based on the gravitational law and Newton's second motion law. In this way, for an $n$-dimensional data set with $N$ data points, each data point is considered as an object in the $n$ dimensional space with mass equal to 1 . Each point in the data set is moved according to a simplified version of the Gravitational Law using the Second Newton's Motion Law. The basic ideas behind applying the gravitational law are:

1) A data point in some cluster exerts a higher gravitational force on a data point in the same cluster than on a data point that is not in the cluster. Then, points in a cluster move in the direction of the center of the cluster. In this way, the proposed technique will determine automatically the clusters in the data set.

2) If some point is a noise point, i.e., does not belong to any cluster, then the gravitational force exerted on it from other points is so small that the point is almost immobile. Therefore, noise points will not be assigned to any cluster.

In order to reduce the amount of memory and a time expended in moving a data point according to the gravitational field generated by another point $(y)$, we use the following simplified equation:

$$
x(t+1)=x(t)+\vec{d} \frac{G}{\|\vec{d}\|^{3}}
$$

where, $\vec{d}=\vec{y}-\vec{x}$, and the gravitational constant $G$.

The velocity at any time, $v(t)$, is considered the zero vector and $\triangle(t)=1$. Since the distance between points is reduced each iteration, all the points will be moved to a single position after a huge (possibly infinite) number of iterations, (big crunch). Then, the gravitational clustering algorithm will define a single cluster. Moreover, the gravitational constant $G$ is reduced each iteration in a constant proportion (the decay term: $\triangle(G)$ ) to eliminate this limit effect. Algorithm 2 shows the randomized gravitational clustering algorithm.

Function Move (line 6), moves both points $x_{j}$ and $x_{k}$ using equation 11 taking into consideration that both points cannot move further than half of the distance between them. In each iteration, RGC creates a set of clusters by using an optimal disjoint set union-find structure ${ }^{3}$ and the distance between

\footnotetext{
${ }^{3} \mathrm{~A}$ disjoint set Union-Find structure is a structure that supports the following three operators [22]:

- MakeSet $(x)$ : Create a new set containing the single element $x$

- $\operatorname{Union}(x, y)$ : Replace the two sets containing $x$ and $y$ by their union.

- $\operatorname{Find}(x)$ : Return the name of the set containing the element $x$
}
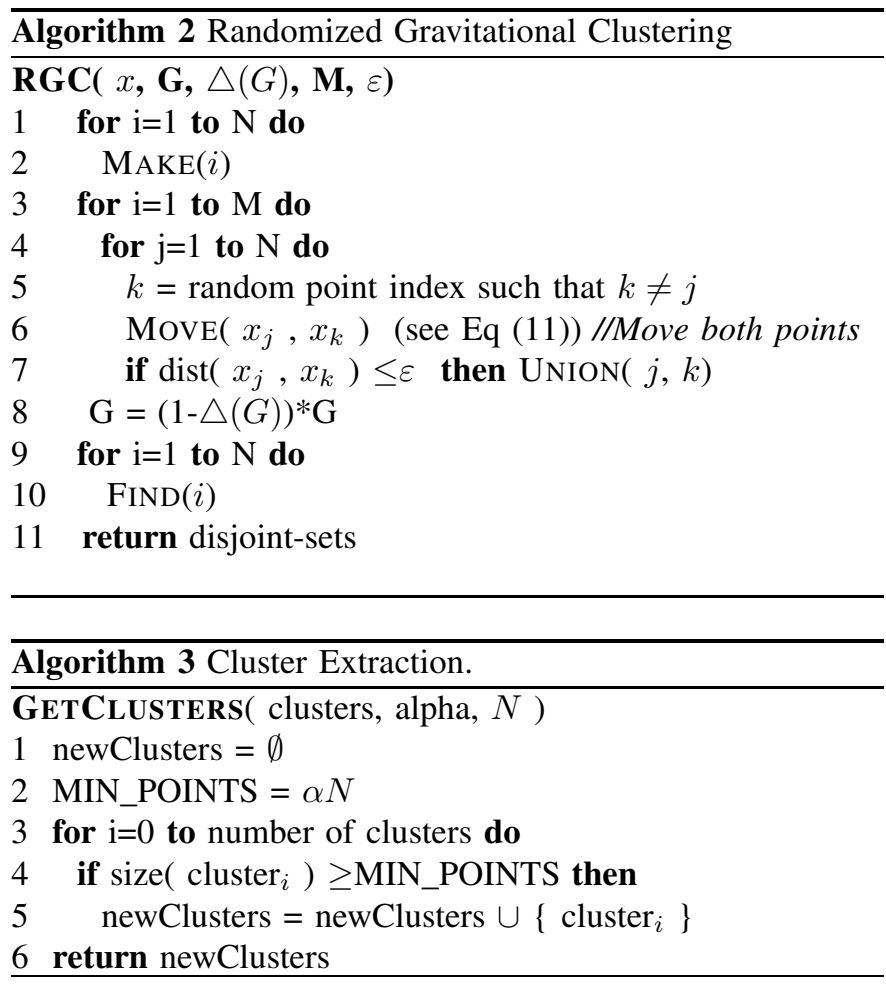

objects (after moving data points according to the gravitational force). When two points are merged, both of them are kept in the system while the associated set structure is modified. In order to determine the new position of each data point, the proposed algorithm only selects another data point in a random way and move both of them according to equation 11 (Move function). RGC returns the sets stored in the disjoint set union-find structure.

Because RGC assigns every point in the data set (noisy or normal) to one cluster, it is necessary to extract the valid clusters. We used an extra parameter $(\alpha)$ to determine the minimum number of points (percentage of the training data set) that a cluster should include in order to be considered a valid cluster. In this way, we used an additional function GetClusters that takes the disjoint sets generated by RGC and returns the collection of clusters that have at least the minimum number of points defined, see Algorithm 3.

RAIN (Clustering based on RAndomized INteractions of data points) extends the RGC algorithm in such a way that different interaction functions can be used (instead of the one based on the Gravitational Law), it reduces the effect of the data set in the dynamics of the system, and it determines automatically the initial interaction strength [REF RAIN].

1) Maximum Distance between Closest Points: In order to reduce the sensitivity of RAIN to the size of the data set, we calculate a rough estimate of the maximum distance between closest points in the data set. This distance will allow RAIN to have a reference value for merging and moving data points.

Given a collection of $N$ data points in the $n$-dimensional

In the optimal disjoint set union-find structure, each set is represented by a tree where the root of the tree is the canonical element of the set, and each child node has a pointer to the parent node (the root node points to itself) $1423^{[2]}$ 
$[0,1]$ Euclidean space, the maximum distance between closest points can be roughly approximate using equation 12 .

$$
\widehat{d}=\frac{2 * \sqrt{n}}{\sqrt{3} * N^{\frac{1}{n}}}
$$

The conjecture behind this equation is that in order to have a maximum distance between closest points, such points should be arranged in a grid defining isosceles triangles (pyramids). The height of such triangle is $\frac{2}{\sqrt{3}}$ the side of the triangle and the maximum number of points per dimension of such grid should be bounded by $N^{\frac{1}{n}} \cdot \sqrt{n}$ is a correction factor for data sets where the number of points is considerably low compared to the number of vertices on the hypercube $[0,1]^{n}$, i.e., $\left(2^{n}\right)$.

2) Moving Functions: Although motivated by the Gravitational Law and second Newton's motion law, RGC can be seen as an algorithm that moves interacting data points according to a decreasing function of the data points distance. In RAIN, the final position of a data point $x$ that is interacting with another data point $y$ is defined by equation 13 .

$$
x(t+1)=x(t)+G * \vec{d} * f\left(\frac{\|\vec{d}\|}{\widehat{d}}\right)
$$

where, $\vec{d}=\vec{y}-\vec{x}$, $f$ is a decreasing function, $\widehat{d}$ is the rough estimate of maximum distance between closest points and $G$ is the initial strength of the data points interaction. Although many decreasing functions can be used, we only consider $f(x)=\frac{1}{x^{3}}$ and $f(x)=e^{-x^{2}}$.

3) Setting the Initial Interaction Strength $(G)$ : Since RAIN is creating the clusters while it is moving the data points, it is possible to use the number of merged points after some checking iterations for determining if RAIN is using an appropriate value of $G$. A point is considered merged if it has been assigned to a cluster of size greater than one. A high number of merged points (more than a half) can indicate that the initial strength is so high that it will define a single big cluster. If the number of merged points is low (less than a half) it can indicate that the initial strength is so low that not cluster is going to be defined at all. Therefore, a value close to the half of the number of data points will indicate an appropriated value for the initial strength. Algorithm 4 shows the process for determining the initial interaction strength.

4) RAIN Time Complexity: Although it looks like RAIN has linear time complexity, our experiments indicates that the number of iterations required is $10 \sqrt{N}$, where $N$ is the number of data points. Also, our experimental results indicate that for checking each candidate initial strength, the number of iterations required is close to $\sqrt{N}$. Therefore, upon experimental evidence the time complexity of RAIN is $O(n \sqrt{n})$.

\section{Fuzzy Self/Non Self Discrimination (T-FD)}

In [23], Gomez proposed an encoding technique that allows an Artificial Immune System approach (AIS) to evolve simple and tuned fuzzy rule detectors based on the mechanism proposed by Gomez in [17] and the extension to the Negative Selection algorithm proposed by Gomez et al in [24]. Instead

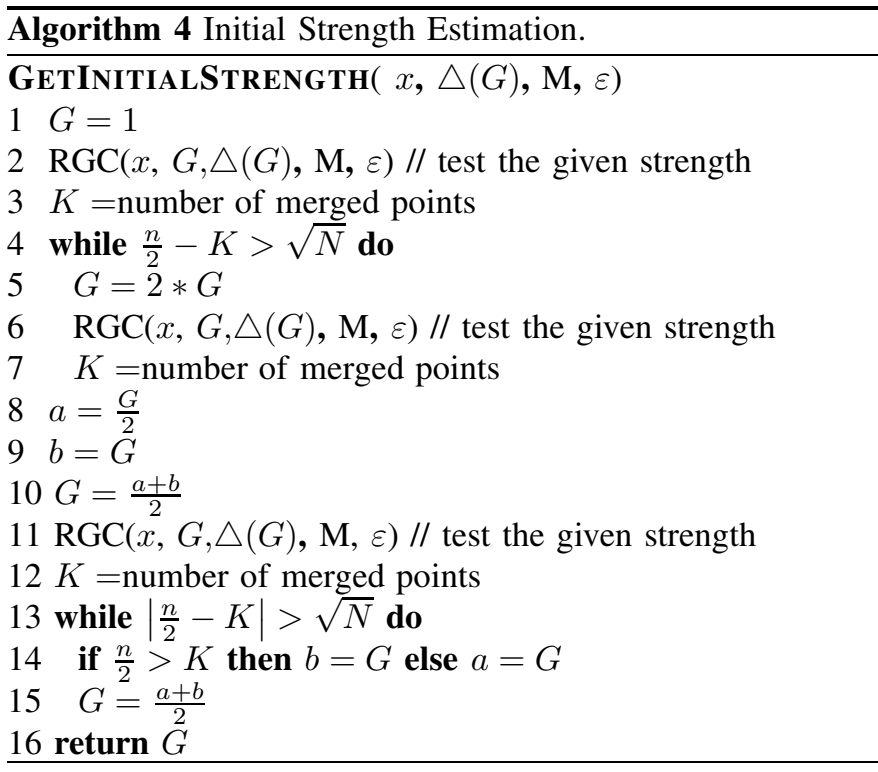

of using a fixed chromosome length, a variable length one is used to evolve the condition part of the fuzzy rule detectors. Such condition is a conjunction of atomic expressions, each one defined in terms of an attribute and a set of parameters that define the fuzzy set.

The idea is to define a fuzzy rule detector as follows:

$$
\text { If } x_{i_{1}} \in T_{i_{1}} \wedge \ldots x_{i_{p}} \in T_{i_{p}} \text { then non_self }
$$

Here, $x=\left(x_{1}, \ldots x_{n}\right)$ is an element of the self/non-self space being evaluated, $\left\{i_{k}\right\}_{k=1, \ldots, p} \subseteq\{1,2, . ., n\}$ is a set of indexes, $T_{i_{k}}$ is fuzzy set defined by a set of parameters, and $\wedge$ is a fuzzy conjunction operator (min-and).

Although Deterministic Crowding ${ }^{4}$ (DC) requires a notion of distance between individuals, it is possible to extend DC in order to use a quasi-metric instead of a distance. A quasimetric has the same properties of a distance but without requiring the symmetry property. Quasi-metrics are useful when comparing sets and rules. Given two sets $s_{1}$ and $s_{2}$, the distance of $s_{1}$ to $s_{2}\left(d\left(s_{1}, s_{2}\right)\right)$ is defined as the proportion of elements of $s_{2}$ that are not in $s_{1}$. In order to extend this definition to fuzzy sets, this quasi-metric can be seen as the area under the membership function of $s_{2}$ that is not contained in the area under membership function of $s_{1}$.

Notice that any fuzzy rule with a variable length condition can be extended in such a way that includes an atomic condition for each attribute: If the condition of a fuzzy rule $R_{1}$ is defined in terms of $k$ atomic expressions, $a_{1}$ AND...AND $a_{k}$, none being defined in terms of attribute $i$, then such a condition is equivalent to the condition $a_{1}$ AND...AND $a_{m}$ AND $x_{i}$ is trapezoid $(0,0,1,1)$. Using these property, the distance from a fuzzy rule $R_{1}$ to a fuzzy rule $R_{2}$ can be defined as the average distance between the fuzzy sets of its attributes to the fuzzy sets of the attributes of rule $R_{2}$, see Algorithm 5 .

It is possible to prove that the distance from fuzzy rule $R_{1}$ to fuzzy rule $R_{2}$ is 0 if the degree of abnormally predicted by

${ }^{4} \mathrm{~A}$ niching technique for Evolutionary algorithms developed by Mahfoud $4^{\text {[25] }}$ 


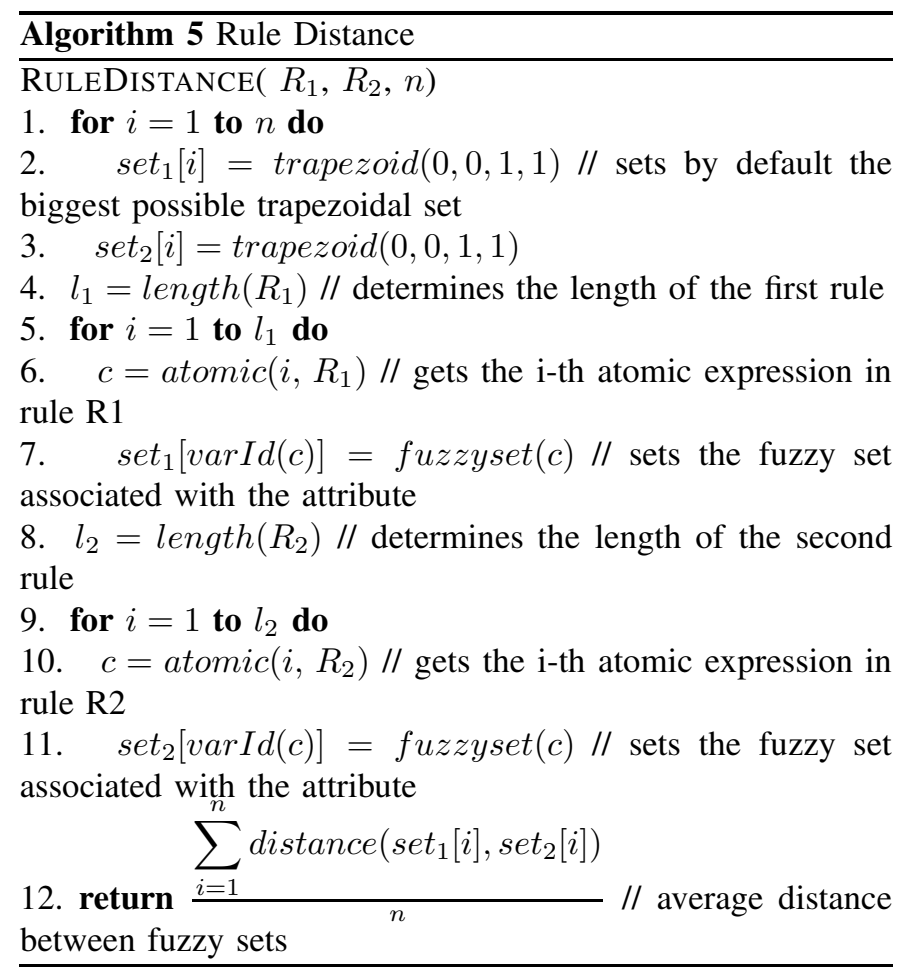

$R_{1}$, for any sample $x$, is higher than the degree of abnormally predicted by $R_{2}$. In other words, anything that $R_{2}$ predicts as abnormal is predicted by $R_{1}$ as abnormal too. It is said that $R_{1}$ dominates $R_{2}$ and $R_{2}$ is dominated by $R_{1}$ when $d\left(R_{1}, R_{2}\right)=0$.

Fuzzy rule detectors evolved by the EA are sorted by fitness from highest to lowest. Detectors with a fitness lower than the population average are removed. The average fitness is a good indicative of the "performance" of the detectors. Then, detectors that are dominated by other detectors with a higher fitness are removed because they are not covering additional non-self space and can cover self space. Finally, detectors that are dominated, regardless their fitness, are removed.

\section{EXPERIMENTATION}

Four different experiments were carried out in order to determine the effectiveness of CORAL:

1) Aggregation of the evolution of fuzzy rules with fuzzy set tuning mechanism (EFR). Each site will have associated an EFR classifier that will discriminate between the positive and negative class.

2) Aggregation of the fuzzy self/non-self discrimination technique with fuzzy set tuning mechanism (T-FD). Each site will have associated a set of fuzzy rule detectors that will discriminate the positive class.

3) Aggregation of the random interaction clustering algorithm (RAIN). Each site will have associated a set of cluster prototypes that will discriminate the negative class.

4) Aggregation of the above three approaches (EFR, TFD, and RAIN). Some sites will have associated an EFR classifier, while others a set of fuzzy rule detectors (T-FD) and the remaining a set of cluster prototypes
(RAIN). This experiment is performed in order to show the integration capability provided by CORAL.

\section{A. Experimental Settings}

Experiments are performed on the two-class machine learning data sets shown in table I.

TABLE I

MACHINE LEARNing Testbed

\begin{tabular}{|c|c|c|c|}
\hline DATA SET & \multirow{2}{*}{ DIM } & \multicolumn{2}{|c|}{ SAMPLES } \\
\cline { 3 - 4 } & & TOTAL & PER CLASS \\
\hline \hline BREAST & 9 & 699 & $\{458,241\}$ \\
\hline PIMA & 8 & 768 & $\{500,268\}$ \\
\hline HEART & 13 & 270 & $\{150,120\}$ \\
\hline
\end{tabular}

Each technique used the parameters proposed in their respective papers. A $3 \times 3$ CORAL lattice is used in all the experiments. An special 10 folding cross-validation technique is applied to each data set. In this way, each of the 9 sites (the classifier associated with such site) is trained with 1 of the 10 groups, and the remaining group is used as testing set. It is done 5 different times. The reported results are the average over those 50 runs. A coupling parameter of $\varepsilon=0.5$ was used in order to give more importance to the site than to its neighbors and the CORAL classifier was iterated by 20 periods of time using the logistic function with parameter $\alpha=1.5$.

Figure 4 shows the distribution of techniques used by CORAL -4 (combination of all techniques).

\begin{tabular}{|c|c|c|}
\hline EFR & RAIN & T-FD \\
\hline RAIN & T-FD & EFR \\
\hline T-FD & EFR & RAIN \\
\hline
\end{tabular}

Fig. 4. Distribution of Techniques used by CORAL-4 (combination of all techniques).

\section{B. Analysis of Results}

Table II shows the performance (accuracy) reached by CORAL after 20 iterations using EFR, T-FD, RAIN, and all of them.

TABLE II

Comparative Performance of Coral

\begin{tabular}{|c|c|c|c|}
\cline { 2 - 4 } \multicolumn{1}{c|}{} & BREAST & HEART & PIMA \\
\hline CORAL-EFR & $97.68 \pm 1.87$ & $92.81 \pm 5.10$ & $77.79 \pm 4.57$ \\
\hline CORAL-T-FD & $97.80 \pm 2.12$ & $69.85 \pm 9.34$ & $67.42 \pm 1.92$ \\
\hline CORAL-RAIN & $83.94 \pm 9.82$ & $61.56 \pm 9.79$ & $65.81 \pm 3.04$ \\
\hline CORAL-AII & $95.74 \pm 2.36$ & $68.30 \pm 6.67$ & $67.51 \pm 2.78$ \\
\hline EFR & $94.85 \pm 2.41$ & $78.96 \pm 8.26$ & $74.21 \pm 5.63$ \\
\hline T-FD & $92.85 \pm 3.61$ & $62.44 \pm 9.04$ & $65.47 \pm 4.66$ \\
\hline RAIN & $87.30 \pm 5.73$ & $62.82 \pm 5.78$ & $65.36 \pm 1.45$ \\
\hline
\end{tabular}

When CORAL is used with EFR, it improves the accuracy of the classifier system in at least $2.8 \%$ for all three data sets. Moreover, for the Heart data set, the accuracy is increased in almost $14 \%$. Similar behavior is observed when Coral is used 25 with T-FD. In this case the accuracy increased in at least $1.9 \%$ 
with an increase around $7 \%$ for the Heart data set. However, this behavior is not observed in the case of CORAL when using RAIN. In this case Coral is better only in the Pima data set for a small margin (less than 0.5\%). For the Breast data set CORAL reduces the accuracy by almost $3.5 \%$. It is possible that RAIN is not able to generate good cluster prototypes from the small amount of information it is training on - each site is trained with $10 \%$ of the data sett meaning around 45 normal (negative) samples.

\section{Experiments with Quantum Physics Data Set}

In order to determine the performance of the proposed approach on real data sets, experiments were conducted on the quantum physics data set introduced in the KDDCup 2004 competition. The training data set was divided in 10 different groups randomly selected. Then, a EFR classifier was trained with nine (9) of those groups. Next, these fuzzy classifiers were organized in a $3 * 3$ CORAL-EFR. Finally, the unused training group was used for testing the CORAL-EFR classifier. Table III reports the results obtained by Coral-EFR and compares it against the average performance of the EFR classifiers. As shown Coral-EFR increases the performance of EFR in almost $3.6 \%$.

TABLE III

Comparative Performance of CoRAL on the QuANTUM Physics DATA SET

\begin{tabular}{|c|c|}
\cline { 2 - 2 } \multicolumn{1}{c|}{} & Performance \\
\hline CORAL-EFR & 68.24 \\
\hline EFR & 64.67 \\
\hline
\end{tabular}

\section{Vi. CONClusions}

An aggregation technique based on Coupled Map Lattices and fuzzy logic was introduced. The results indicate that it improves the accuracy and robustness of the classifier while reducing the training time. It was shown that several different training classification techniques can be used and integrated into a CORAL classification technique.

\section{REFERENCES}

[1] J. Han and M. Kamber, Data Mining: Concepts and Techniques. Morgan Kaufmann, 2000.

[2] R. S. Michalski, I. Bratko, and M. Kubat, Machine Learning and Data Mining: Methods and Applications. J. Wiley \& Sons, 1998.

[3] R. Holte, "Very simple classification rules perform well in most common used datasets," Machine Learning, no. 11, pp. 63-91, 1993.

[4] G. Valentini and F. Masulli, "Ensembles of learning machines," in Proceedings of the 13th Italian Workshop on Neural Nets-Revised Papers, pp. 3-22, 2002.

[5] K. Kaneko, Theory and Applications of Couple Map Lattices. John Wiley, 1993.

[6] R. Kozma, "Intermediate-range coupling generates low-dimensional attractors in the chaotic region of one-dimensional lattices," Physics Letters A, no. 244, pp. 85-91, 1998.

[7] S. Wolfram, A New Kind of Science. Wolfram Media, 2002.

[8] J. Gomez, D. Dasgupta, O. Nasraoui, and F. Gonzalez, "Complete expression trees for evolving fuzzy classifier systems with genetic algorithms," in Proceedings of the North American Fuzzy Information Processing Society Conference NAFIPS-FLINTS 2002, pp. 469-474, 2002.
[9] M. V. Fidelis, H. S. Lopes, and A. A. Freitas, "Discovering comprehensible classification rules with a genetic algorithm," in Proceedings of Congress on Evolutionary Computation (CEC), pp. 805-810, 2000.

[10] H. Ishibushi and T. Nakashima, "Liguistic rule extraction by geneticsbased machine learning," in Proceedings of the Genetic and Evolutionary Computation Conference GECCO'O0, pp. 195-202, 2000.

[11] J. Liu and J. Kwok, "An extended genetic rule genetic algorithm," in Proceedings of Congress on Evolutionary Computation (CEC), pp. 458263, 2000.

[12] A. Gonzalez and R. Prez, "Completeness and consistency conditions for learning fuzzy rules," Fuzzy Sets and Systems, no. 96, pp. 37-51, 1998.

[13] K. De Jong and W. Spears, "Learning concept classification rules using genetic algorithms," in Proceedings of the Twelfth International Joint Conference on Artificial Intelligence, pp. 651-656, 1991.

[14] O. Cordon, A. Gonzalez, F. Herrera, and R. Perez, "Encouraging cooperation in the genetic iterative rule learning approach for quality modeling," in Computing with Words in Intelligent/Information Systems 2. Applications, J. Kacprzyk, L. Zadeh (Eds.), Physica-Verlag, 1998.

[15] O. Cordon and F. Herrera, "A general study on genetic fuzzy system," in Genetic Algorithms in Engineering and Computer Sciences, pp. 33-57, Jonh Wiley and Sons, 1995.

[16] A. A. Freitas, "A survey of evolutionary algorithms for data mining and knowledge discovering," in Advances in Evolutionary Computation. A. Ghosh and S. Tsutsui. (Eds.), Springer-Verlag, 2001.

[17] J. Gomez, "Evolution of fuzzy rule based classifiers," in Proceedings of the Genetic and Evolutionary Computation Conference GECCO 2004, 2004.

[18] J. Gomez, "Self adaptation of operator rates in evolutionary algorithms," in Proceedings of the Genetic and Evolutionary Computation Conference (GECCO 2004), June 2004.

[19] C. Karr, "Genetic algorithms for fuzzy controllers," AI Experts, pp. 2633, 1991.

[20] J. Gomez and D. Dasgupta, "Evolving fuzzy rules for intrusion detection," in Proceedings of the Third Annual IEEE Information Assurance Workshop 2002 Conference, pp. 68-75, 2002.

[21] J. Gomez, D. Dasgupta, and O. Nasraoui, "A new gravitational clustering algorithm," in Proceedings of the Third SIAM International Conference on Data Mining 2003, 2003.

[22] T. Cormer, C. Leiserson, and R. Rivest, Introduction to Algorithms. McGraw Hill, 1990.

[23] J. Gomez, Soft Computing Techniques for Intrusion Detection. PhD thesis, The University of Memphis, 2004.

[24] J. Gomez, F. Gonzalez, M. Kaniganti, and D. Dasgupta, "An evolutionary approach to generate fuzzy anomaly (attack) signatures," in Proceedings of the IEEE Information Assurance Workshop 2003 Conference, 2003.

[25] S. W. Mahfoud, "Crowding and preselection revisited," in Proceedings Second Conference Parallel Problem Solving from Nature, 1992. 